\title{
Some results on quantile-based dynamic survival and failure Tsallis entropy
}

\author{
Rekha Rani, Vikas Kumar and Nirdesh Singh
}

\begin{abstract}
Non-additive entropy measures are important for many applications. In this paper, we introduce a quantile-based non-additive entropy measure, based on Tsallis entropy and study their properties. Some relationships of this measure with well-known reliability measures and ageing classes are studied and some characterization results are presented. Also the concept of quantile-based shift independent entropy measures has been introduced and studied various properties.
\end{abstract}

Keywords. Tsallis entropy, cumulative residual entropy, weighted entropy, quantile function, reliability measures

\section{Introduction}

It is very much common in machine learning to quantify the expected amount of information associated with stochastic events, and to quantify the similarity between probability distributions. In both cases, entropy is used as a measure for information content of probability distributions. To measure the uncertainty associated with a probability distribution, Shannon [34] proposed a measure called Shannon entropy. Here and throughout the article, $X$ is absolutely continuous nonnegative random variable with probability density function $f(x)$, the Shannon entropy is defined as,

$$
H(X)=-\int_{0}^{\infty} f(x) \log f(x) d x
$$

The characteristic property of Shannon entropy is additivity, in the sense that for two independent random variables $X$ and $Y$

$$
H(X * Y)=H(X)+H(Y),
$$

where $X * Y$ denotes the joint random variable. If a unit is known to have survived up to an age $t$, then (1.1) is not applicable. Accordingly, Ebrahimi [8] considered the entropy of the residual lifetime $X_{t}=[X-t \mid X>t]$ as a dynamic version of uncertainty given by

$$
H(X ; t)=-\int_{t}^{\infty} \frac{f(x)}{\bar{F}(t)} \log \frac{f(x)}{\bar{F}(t)} d x, t \geq 0 .
$$

Received date: October 7, 2020; Published online: March 16, 2021.

2010 Mathematics Subject Classification. 62N05; 90B25.

Corresponding author: Vikas Kumar. 
A well known generalization of Shannon entropy is Tsallis entropy that was first introduced by Havrda and Charvat [12] in the context of cybernetics theory. Then, Tsallis [31] exploited its non-extensive features and placed it in a physical setting. This measure is defined as

$$
H_{\alpha}(X)=\frac{1}{(1-\alpha)}\left(\int_{0}^{\infty} f^{\alpha}(x) d x-1\right), \alpha>0, \alpha \neq 1 .
$$

As $\alpha \longrightarrow 1,(1.3)$ reduces to (1.1). Generalizing the concept of Ebrahimi [8], Asadi et al. [2] and Kumar and Taneja [20] presented Tsallis entropy of residual lifetime and studied some properties of it. Moreover, the Tsallis entropy is a non-additive entropy as for any two independent random variables $X$ and $Y$

$$
H_{\alpha}(X * Y)=H_{\alpha}(X)+H_{\alpha}(Y)+(1-\alpha) H_{\alpha}(X) H_{\alpha}(Y) .
$$

Extensive or non-extensive statistical mechanics arise from the additivity or non-additivity of the corresponding entropy measures. The concept of Tsallis entropy provides an extension of thermodynamics and statistical physics. In physics, Tsallis entropy is used to describe a number of non-extensive systems (Hamity and Barraco, [10], image processing (Yu et al., [39] and signal processing (Tong et al., [30]. In the ecology, Tsallis entropy is proposed to a new class of diversity indicies which covers many common diversity indicies found in ecological literature. Also, the Tsallis entropy has been applied to a wide spectrum of problems in water engineering.

Rao et al. [28] have pointed out some basic shortcomings of the Shannon differential entropy, and defined a new measure of uncertainty based on the survival function of a random variable $X$ instead of its density function, as

$$
\xi(X)=-\int_{0}^{\infty} \bar{F}(x) \log \bar{F}(x) d x
$$

and called it cumulative residual entropy (CRE). CRE is always nonnegative; however $H(X)$ and $H_{\alpha}(X)$ are nonnegative for discrete random variables and can be negative in a continuous case. Some properties and applications of CRE in reliability engineering and computer vision have been studied by Rao [29] and Wang and Vemuri [38]. Asadi and Zohrevand [3] have considered the dynamic version of $C R E$, which is defined as

$$
\xi(X ; t)=-\int_{t}^{\infty} \frac{\bar{F}(x)}{\bar{F}(t)} \log \frac{\bar{F}(x)}{\bar{F}(t)} d x, t \geq 0 .
$$

There have been attempts by several authors for the parametric generalization of CRE. The alternate form for Tsallis entropy proposed by Rajesh and Sunoj [27] based on the survival entropy (SE) of order $\alpha$ is given as

$$
\xi_{\alpha}(X)=\frac{1}{(1-\alpha)}\left(\int_{0}^{\infty} \bar{F}^{\alpha}(x) d x-E(X)\right), \alpha>0, \alpha \neq 1,
$$

and called it the cumulative residual Tsallis entropy (CRTE). Measure (1.6) approaches the CRE as $\alpha \longrightarrow 1$. Also, they have considered the dynamic version of CRTE, which is defined as

$$
\xi_{\alpha}(X ; t)=\frac{1}{(1-\alpha)}\left(\frac{\int_{t}^{\infty} \bar{F}^{\alpha}(x) d x}{\bar{F}^{\alpha}(t)}-E\left(X_{t}\right)\right),
$$

and called it the dynamic cumulative residual Tsallis entropy (DCRTE). For more properties and applications of these information measures we refer to Abbasnejad et al. [1] and Kumar [17]. 
All the theoretical investigations and applications using these information measures are based on the distribution function, but may not be suitable in situations where the distribution is not analytically tractable. An alternative approach to the study is to use the quantile function ( $Q F)$, defined by

$$
Q(u)=F^{-1}(u)=\inf \{x \mid F(x) \geq u\}, \quad 0 \leq u \leq 1 .
$$

Quantile functions (QFs) have several properties that are not shared by distribution functions, refer to Gilchrist [9] and Nair et al. [23]. Quantile functions can be properly employed to formulate properties of entropy function and other information measures. The study of entropy functions using quantile functions is of recent interest. Quantile-based information measures have been used by many researchers in the context of information theory. We refer to the reader, Sunoj and Sankaran [35], Sunoj et al. [37], Nanda et al. [26], Kumar and Rekha [18], Kayal and Tripathy [14] and Krishnan et al. [16] and many others.

Motivated by these in the present study we consider the quantile-based dynamic (residual and past, both) cumulative Tsallis non-additive entropy measure, since non-additivity rather than additivity is more prevalent in many physical situations. It has been indicated that the use of different entropy measures may lead to different models or statistical results than those obtained by Shannon and Kullback-Leibler measures.

The text is organized as follows. In Section 2, we introduce the quantile-based cumulative residual Tsallis entropy (CRTE) in residual lifetime and various properties of the measure are discussed. Section 3 proves some characterization results based on the measure considered in Section 2. In Section 4, the proposed quantile measure is used to study two ageing classes of distribution. In Section 5, the quantile-based cumulative Tsallis entropy (CTE) in reversed residual (past) lifetime are discussed. In Section 6, we derive the weighted form of this measure and call it weighted cumulative residual Tsallis quantile entropy (WCRTQE) and study some characterization results.

\section{Quantile-based Cumulative Residual Tsallis Entropy}

We assume that $X$ is a nonnegative random variable with absolutely continuous distribution function $F(x)$ and probability density function $f(x)$. When $F(x)$ is strictly increasing, the quantile function $Q(u)$ is the solution of $F(x)=u$ as $x=Q(u)$. The mean of the distribution assumed to be finite, is

$$
E(X)=\int_{0}^{1} Q(p) d p=\int_{0}^{1}(1-p) q(p) d p
$$

where $q(u)=\frac{d Q(u)}{d u}$ is the quantile density function. Defining the density quantile function by $f Q(u)=f(Q(u))$ and the quantile density function by $q(u)$, we have

$$
q(u) f Q(u)=1 \text {. }
$$

The hazard quantile function defined by

$$
K(u)=h(Q(u))=\frac{f Q(u)}{(1-u)}=\frac{1}{(1-u) q(u)},
$$

where $h(x)=\frac{f(x)}{1-F(x)}$ is the hazard rate of $X$. Another useful measure closely related to hazard $\mathrm{QF}$ is the mean residual quantile function, as given by

$$
M(u)=m(Q(u))=(1-u)^{-1} \int_{u}^{1}(1-p) q(p) d p,
$$


where $m(t)=E(X-t \mid X>t)$ is the mean residual life function (MRLF) of $X$. It is well known that both hazard quantile function and mean residual quantile function uniquely determine the quantile density function $q(u)$. Further the relationship between the quantile density function and mean residual quantile function is given by

$$
q(u)=\frac{M(u)-(1-u) M^{\prime}(u)}{(1-u)} .
$$

Recently, Sankaran and Sunoj (2017) have introduced the quantile versions of the cumulative residual entropy (6.1) and its dynamic form (1.5). The quantile-based dynamic cumulative residual entropy (DCRE) is defined by

$$
\xi(u)=\xi(X ; Q(u))=\frac{\log (1-u)}{(1-u)} \int_{u}^{1}(1-p) q(p) d p-(1-u)^{-1} \int_{u}^{1} \log (1-p)(1-p) q(p) d p .
$$

When $u \longrightarrow 0,(2.5)$ reduces to $\xi=-\int_{0}^{1}(\log (1-p))(1-p) q(p) d p$, a quantile version of $C R E$.

Following with Sankaran and Sunoj [32], the quantile version of cumulative residual Tsallis entropy (CRTE) of the nonnegative random variable $X$ is defined as

$$
\xi_{\alpha}=\frac{1}{(1-\alpha)}\left(\int_{0}^{1}(1-p)^{\alpha} q(p) d p-\int_{0}^{1}(1-p) q(p) d p\right) .
$$

There are some models that do not have any closed form expressions for distribution and density function, but have simple QFs or quantile density functions.

Example 1. A lambda family of distribution that is of interest in reliability is the Davis distribution proposed by Hankin and Lee [11] with quantile function

$$
Q(u)=C u^{\lambda_{1}}(1-u)^{-\lambda_{2}}, C, \lambda_{1}, \lambda_{2} \geq 0 \geq ; 0 \leq u \leq 1 .
$$

This is a flexible family for right skewed nonnegative data that provides good approximations to the exponential, gamma, lognormal, and Weibull distributions. A special feature of these families is that they are expressed in terms of QFs for which distribution functions are not available in closed form to facilitate the conventional analysis.

The cumulative residual Tsallis quantile entropy (CRTQE) (2.6), for Davis (power-Pareto) distribution is given as

$$
\begin{aligned}
\xi_{\alpha}=\frac{C}{(1-\alpha)}[ & \lambda_{1}\left\{\beta\left(\lambda_{1}, \alpha-\lambda_{2}+1\right)-\beta\left(\lambda_{1}, 2-\lambda_{2}\right)\right\} \\
& \left.+\lambda_{2}\left\{\beta\left(\lambda_{1}+1, \alpha-\lambda_{2}\right)-\beta\left(\lambda_{1}+1,1-\lambda_{2}\right)\right\}\right] .
\end{aligned}
$$

As $\lambda_{2} \longrightarrow 0,(2.7)$ reduces to $\xi_{\alpha}=\frac{C \lambda_{1}}{(1-\alpha)}\left(\beta\left(\lambda_{1}, 1+\alpha\right)-\frac{1}{\lambda_{1}\left(1+\lambda_{1}\right)}\right)$, corresponding to the power distribution. Also as $\lambda_{1} \longrightarrow 0,(2.7)$ reduces to $\xi_{\alpha}=\frac{C \lambda_{2}}{\left(1-\lambda_{2}\right)\left(\alpha-\lambda_{2}\right)}$, corresponding to the Pareto II distribution.

Example 2. If $X$ is a random variable following the Govindarajulu's distribution (1977) with the quantile fuction $Q(u)=a\left\{(b+1) u^{b}-b u^{b+1}\right\}, 0 \leq u \leq 1 ; a, b>0$, then $\xi_{\alpha}$ becomes $\xi_{\alpha}=$ $\frac{a b(b+1)}{(1-\alpha)}[\beta(\alpha+2, b)-\beta(b, 3)]$. 
An equivalent definition for the dynamic cumulative residual Tsallis entropy (DCRTE) in terms of $\mathrm{QF}$ is given by

$$
\begin{aligned}
\xi_{\alpha}(u) & =\xi_{\alpha}(X ; Q(u))=\frac{1}{(1-\alpha)}\left(\int_{u}^{1} \frac{(\bar{F}(Q(p)))^{\alpha} q(p) d p}{(1-u)^{\alpha}}-\int_{u}^{1} \frac{(\bar{F}(Q(p))) q(p) d p}{(1-u)}\right), \\
= & \frac{1}{(1-\alpha)}\left(\frac{1}{(1-u)^{\alpha}} \int_{u}^{1}(1-p)^{\alpha} q(p) d p-\frac{1}{(1-u)} \int_{u}^{1}(1-p) q(p) d p\right) .
\end{aligned}
$$

The measure (2.8) may be considered as the dynamic cumulative residual Tsallis quantile entropy (DCRTQE) measure. When $\alpha \longrightarrow 1$, the measure (2.8) reduces to (2.5). Equation (2.8) can be written as

$$
\begin{aligned}
& (1-\alpha) \xi_{\alpha}(u)=\frac{1}{(1-u)^{\alpha}} \int_{u}^{1}(1-p)^{\alpha} q(p) d p-M(u) . \\
& (1-u)^{\alpha}\left((1-\alpha) \xi_{\alpha}(u)+M(u)\right)=\int_{u}^{1} \frac{(1-p)^{\alpha-1}}{K(p)} d p,
\end{aligned}
$$

where (2.9) and (2.10) are the expressions of $\xi_{\alpha}(u)$ in terms of the mean residual quantile function $M(u)$ and hazard quantile function $K(u)$, respectively. Using (2.4), Equation (2.9) can be written as

$$
(1-u)^{\alpha}\left((1-\alpha) \xi_{\alpha}(u)+M(u)\right)=\int_{u}^{1}(1-p)^{\alpha-1} M(p) d p-\int_{u}^{1}(1-p)^{\alpha} M^{\prime}(p) d p
$$

Applying integration by parts on the last term and simplify, we obtain

$$
(1-u)^{\alpha} \xi_{\alpha}(u)=\int_{u}^{1}(1-p)^{\alpha-1} M(p) d p .
$$

Differentiating (2.9) with respect to $u$ both sides, and using (2.4) reduces to

$$
M(u)=\alpha \xi_{\alpha}(u)-(1-u) \xi_{\alpha}^{\prime}(u) .
$$

Since Tsallis entropy for residual lifetime does not determines distribution function uniquely, refer to Nanda and Paul [25]. However Equations (2.12) and (2.13) provide a direct relationship between mean residual quantile function $M(u)$ and $\xi_{\alpha}(u)$, which show that $\xi_{\alpha}(u)$ uniquely determines the quantile density function $q(u)$.

Remark 1. When $\alpha \longrightarrow 1$ then (2.12) reduces to

$$
(1-u) \xi(u)=\int_{u}^{1} M(p) d p
$$

a result obtained by Sankaran and Sunoj [32].

The next theorem gives necessary and sufficient conditions for $\xi_{\alpha}(u)$ to be an increasing (decreasing) function of $u$. In this context, we state the following theorem without proof.

Theorem 2.1. Let $X$ be a nonnegative absolutely continuous random variable having survival function $\bar{F}(x)$, then $\xi_{\alpha}(u)$ is increasing (decreasing), if and only if for $u \geq 0$

$$
\xi_{\alpha}(u) \geq(\leq) \frac{M(u)}{\alpha} .
$$

We observe that, the monotone behavior of $\xi_{\alpha}(u)$ purely depends on the mean residual quantile function $M(u)$. For some univariate continuous distributions, the expression (2.3) is evaluated as given below in Table 2.1. It is clear from the Table 2.1 that for uniform distribution $\xi_{\alpha}(u)=$ $\frac{M(u)}{\alpha+1}$ and thus it belongs to the decreasing dynamic cumulative residual Tsallis quantile entropy (DDCRTQE). 
Table 2.1 Mean residual quantile function $M(u)$ and DCRTQE $\xi_{\alpha}(u)$ for some lifetime distributions

\begin{tabular}{|c|c|c|c|}
\hline Distribution & Quantile function $Q(u)$ & $\mathrm{M}(\mathrm{u})$ & $\xi_{\alpha}(u)$ \\
\hline Uniform & $a+(b-a) u$ & $\frac{(b-a)(1-u)}{2}$ & $\frac{(b-a)(1-u)}{2(\alpha+1)}$ \\
Exponential & $-\lambda^{-1} \log (1-u)$ & $\frac{1}{\alpha}$ & $\frac{1}{\alpha \lambda}$ \\
Gompertz & $\frac{1}{\log c}\left(1-\frac{\log c \log (1-u)}{B}\right)$ & $\frac{1}{B}$ & $\frac{a b(1-u)^{-\frac{1}{a}}}{(a-1)(a \alpha-1)}$ \\
Pareto II & $a\left[(1-u)^{-\frac{1}{b}}-1\right]$ & $\frac{b(1-u)^{-\frac{1}{a}}}{a-1}$ & $\left(\frac{b(a+1)(1-u)^{-\frac{a}{a+1}}}{\alpha a+\alpha-a}\right)$ \\
Generalized Pareto & $\frac{b}{a}\left[(1-u)^{-\frac{a}{a+1}}-1\right]$ & $b(1-u)^{-\frac{a}{a+1}}$ & $\frac{a b(1-u)^{\frac{1}{a}}}{(a+1)(a \alpha+1)}$ \\
Finite Range & $b\left(1-(1-u)^{\frac{1}{a}}\right)$ & $\frac{b(1-u)^{\frac{1}{a}}}{a+1}$ & $\left.\frac{\bar{\beta}}{b\left(\frac{1}{b}, \alpha-\frac{1}{b}\right)}-\frac{\bar{\beta}_{u}\left(\frac{1}{b}, 1-\frac{1}{b}\right)}{(1-u)}\right]$ \\
Log-Logestic & $\frac{1}{a}\left(\frac{u}{(1-u)}\right)^{\frac{1}{b}}$ & $\frac{\bar{\beta}_{u}\left(\frac{1}{b}, 1-\frac{1}{b}\right)}{a b(1-u)}$ & $\frac{1}{(1-\alpha) a b}\left[\frac{(a-u)^{\alpha}}{\alpha}-\frac{2(1-u)}{\alpha+1}\right.$ \\
Linear Mean & $-(a+b) \ln (1-u)-4 u$ & $-(a+b+2)+2 u$ & $-\frac{(a+b)}{\alpha}$ \\
Residual Quantile &
\end{tabular}

\section{Characterization of Lifetime Distribution Functions}

By considering a relationship between the dynamic cumulative residual Tsallis quantile entropy $\xi_{\alpha}(u)$ and the mean residual quantile function $M(u)$, we characterize some lifetime distributions based on the quanlile entropy measure (2.8). We give the following theorem.

Theorem 3.1. Let $X$ be a nonnegative continuous random variable with quantile function $Q(u)$ and mean residual quantile function $M(u)$. The relationship

$$
\xi_{\alpha}(u)=c M(u)
$$

where $c=\left(\frac{a+1}{\alpha(a+1)-a}\right)$ is constant, holds for all $u$, if and only if, $X$ has generalized Pareto distribution with quantile function

$$
Q(u)=\frac{b}{a}\left[(1-u)^{-\frac{a}{a+1}}-1\right] ; b>0, a>-1 .
$$

Proof. The mean residual quantile function of generalized Pareto distribution is $M(u)=b(1-$ $u)^{-\frac{a}{a+1}}$. Taking $c=\left(\frac{a+1}{\alpha(a+1)-a}\right)$ gives the if part of the theorem. To prove the only if part, consider (3.1) to be valid. Using (2.13) in equation (3.1) and simplifying, we get

$$
\frac{M^{\prime}(u)}{M(u)}=\frac{(c \alpha-1)}{c(1-u)}
$$

Integrate it with respect to $u$ both sides, we get

$$
M(u)=A(1-u)^{-\left(\alpha-\frac{1}{c}\right)} .
$$

Substituting the value of $c$, this gives

$$
M(u)=A(1-u)^{-\frac{a}{a+1}},
$$

which characterizes the generalized Pareto distribution. Hence proved. 
Corollary 3.2. Let $X$ be a nonnegative continuous random variable with quantile function $Q(u)$ and mean residual quantile function $M(u)$. The relationship

$$
\xi_{\alpha}(u)=c M(u)
$$

holds for all $u$, if and only if, for (i) $c=\frac{1}{\alpha}, X$ has exponential distribution (ii) $c<\frac{1}{\alpha}$, $X$ has Pareto II distribution (iii) $c>\frac{1}{\alpha}, X$ has finite range distribution.

Next, we extend the result (3.1) given by Theorem 3.1 to a more general case. We state the following result which is easy to prove, and hence omitted.

Theorem 3.3. Let $X$ be a nonnegative continuous random variable with mean residual quantile function $M(u)$ and DCRTQE $\xi_{\alpha}(u)$ given by

$$
\xi_{\alpha}(u)=c(u) M(u) \text { for } u \geq 0,
$$

then

$$
M(u)=\frac{\exp \left(-\int_{u}^{1} \frac{d u}{(1-u) c(u)}\right)}{c(u)(1-u)^{\alpha}} .
$$

In particular if $c(u)=a u+b$, and $a, b \geq 0$, then (3.3) gives

$$
M(u)=\frac{1}{(a u+b)(1-u)^{\alpha}}\left(\frac{(1-u)(a+b)}{(a u+b)}\right)^{\frac{1}{a+b}} .
$$

Further we note that the expression (3.4) for $a=0$, gives the characterization result given by the Corollary 3.2.

\section{Ageing Classes Based on DCTRQE}

The notion of ageing plays an important role in reliability analysis and in identifying life distribution. One of the objectives of ageing concepts in the distribution function approach to quantile forms is to analyse lifetime data using quantile functions which do not have tractable distribution function. Based on the dynamic cumulative Tsallis residual quantile entropy (DCTRQE) function, we define the following ageing classes.

Definition 1. A nonnegative random variable $X$ is said to have decreasing (increasing) dynamic cumulative residual Tsallis quantile entropy (DDCRTQE (IDCRTQE)) if $\xi_{\alpha}(u)$ is decreasing (increasing) in $u \geq 0$.

The following theorem show that exponential distribution is the only distribution which is both DDCRTQE and IDCRTQE.

Theorem 4.1. If a distribution is DDCRTQE as well as IDCRTQE for some constant $\alpha$, then it must be exponential.

Proof. $X$ is DDCRTQE means

$$
\frac{1}{1-\alpha}\left(\int_{u}^{1} \frac{(1-p)^{\alpha} q(p) d p}{(1-u)^{\alpha}}-\int_{u}^{1} \frac{(1-p) q(p) d p}{(1-u)}\right) \text { is decreasing in } u \text {. }
$$


$X$ is IDCRTQE means

$$
\frac{1}{1-\alpha}\left(\int_{u}^{1} \frac{(1-p)^{\alpha} q(p) d p}{(1-u)^{\alpha}}-\int_{u}^{1} \frac{(1-p) q(p) d p}{(1-u)}\right) \text { is increasing in } u .
$$

If $X$ satisfies (4.1) and (4.2) both, we must have

$$
\frac{1}{1-\alpha}\left(\int_{u}^{1} \frac{(1-p)^{\alpha} q(p) d p}{(1-u)^{\alpha}}-\int_{u}^{1} \frac{(1-p) q(p) d p}{(1-u)}\right)=\text { Constant. }
$$

This is equivalent to saying that

$$
\int_{u}^{1}(1-p)^{\alpha} q(p) d p=[a(1-\alpha)+M(u)](1-u)^{\alpha},
$$

where $a$ is constant. Differentiating both sides of the above expression with respect to $u$, we get $M(u)=$ constant, which means that the distribution is exponential. The theorem is proved.

Below we see how the ageing classes are affected by increasing transformation. Before the main result we state the following lemma by Nanda et al. [26] which is easy to prove, and hence omitted.

Lemma 4.1. Let $f(u, x): \Re_{+}^{2} \longrightarrow \Re_{+}$and $g: \Re_{+} \longrightarrow \Re_{+}$be any two functions. If $\int_{u}^{\infty} f(u, x) d x$ is increasing and $g(u)$ is increasing (decreasing) in $u$, then $\int_{u}^{\infty} f(u, x) g(x) d x$ is increasing (decreasing) in u, provided the integrals exist.

Theorem 4.2. Let $X$ be a nonnegative and continuous random variable with quantile function $Q_{X}(u)$ and quantile density function $q_{X}(u)$. Define $Y=\phi(X)$, where $\phi($.$) is a nonnegative,$ increasing and convex (concave) function.

(i) For $0<\alpha<1, \xi_{\alpha}\left(Y ; Q_{Y}(u)\right)$ is increasing (decreasing) in u whenever $\xi_{\alpha}\left(X ; Q_{X}(u)\right)$ is increasing (decreasing) in $u$.

(ii) For $\alpha>1, \xi_{\alpha}\left(Y ; Q_{Y}(u)\right)$ is decreasing (increasing) in u whenever $\xi_{\alpha}\left(X ; Q_{X}(u)\right)$ is increasing (decreasing) in $u$.

Proof. (i) The probability density function of $Y=\phi(X)$ is $g(y)=\frac{f\left(\phi^{-1}(y)\right)}{\phi^{\prime}\left(\phi^{-1}(y)\right)}$; hence density quantile funtion is $g\left(Q_{Y}(u)\right)=\frac{1}{q_{Y}(u)}=\frac{f\left(Q_{X}(u)\right)}{\phi^{\prime}\left(Q_{X}(u)\right)}=\frac{1}{q_{X}(u) \phi^{\prime}\left(Q_{X}(u)\right)}$. Thus we have

$$
\begin{aligned}
\xi_{\alpha}\left(Y ; Q_{Y}(u)\right) & =\frac{1}{1-\alpha}\left(\int_{u}^{1} \frac{(1-p)^{\alpha} q_{Y}(p) d p}{(1-u)^{\alpha}}-\int_{u}^{1} \frac{(1-p) q_{Y}(p) d p}{(1-u)}\right) \\
& =\frac{1}{1-\alpha}\left(\int_{u}^{1} \frac{(1-p)^{\alpha} q_{X}(p) \phi^{\prime}\left(Q_{X}(p)\right) d p}{(1-u)^{\alpha}}-\int_{u}^{1} \frac{(1-p) q_{X}(p) \phi^{\prime}\left(Q_{X}(p)\right) d p}{(1-u)}\right) .
\end{aligned}
$$

Since $\phi$ is non negative, increasing convex, we have $\phi^{\prime}\left(Q_{X}(p)\right)$ is increasing and is non negative. Moreover, by the assumption $X$ is IDCRTQE. Hence by Lemma 4.1, (4.3) is increasing. This proves (i). The proof of part (ii) is similar.

The following example illustrates the utility of Theorem 4.2. 
Example 3. Let $X$ be nonnegative random variable with exponential distribution with quantile function $Q(u)=\lambda^{-1}(-\log (1-u)), \lambda>0$, and let $Y=X^{\frac{1}{\beta}}, \beta>0$. Then $Y$ has Weibull distribution with $Q(u)=\lambda^{\frac{-1}{\beta}}(-\log (1-u))^{\frac{1}{\beta}}$. The nonnegative increasing function $\phi(X)=X^{\frac{1}{\beta}}$, is convex (concave) if $0<\beta<1(\beta>1)$ and is nonnegative. Then, by Theorem 4.2 , for $0<\alpha<1$ and $0<\beta<1$ the Weibull distribution is IDCRTQE. For $\alpha>1, \beta>1$, Weibull distribution is DDCRTQE.

\section{Quantile Based Cumulative Tsallis entropy for Inactivity time}

Suppose at time $t$, one has under gone a medical test to check for a certain disease. Let us assume that the test is positive. If we denote by $X$ the age when the patient was infected, then it is known that $X<t$. Now the question is, how much time has elapsed since the patient had been infected by this disease. In this situation, the random variable $\tilde{X}_{t}=[t-X \mid X \leq t]$, which is known as inactivity time is suitable to describe the time elapsed between the failure of a system and the time when it is found to be "down".

The past lifetime random variable $\tilde{X}_{t}$ is related with two relevant ageing functions, the reversed hazard rate defined by $\mu_{F}(x)=\frac{f(x)}{F(x)}$, and mean inactivity time (MIT) defined by $m(t)=E(t-X \mid X<t)=\frac{1}{F(t)} \int_{0}^{t} F(x) d x$. The quantile versions of reversed hazard rate function and mean inactivity time (MIT) are given as

$$
\bar{K}(u)=\bar{K}(Q(u))=u^{-1} f(Q(u))=[u q(u)]^{-1},
$$

and

$$
\bar{M}(u)=m(Q(u))=u^{-1} \int_{0}^{u}[Q(u)-Q(p)] d p=\frac{1}{u} \int_{0}^{u} p q(p) d p,
$$

respectively. The relationship between quantile density function $q(u)$ and mean inactivity quantile function $\bar{M}(u)$ are related as follows

$$
q(u)=\frac{\bar{M}(u)+u \bar{M}(u)}{u},
$$

refer to Nair and Sankaran [24]. Di Crescenzo and Longobardi [7] introduced a dual measure based on the cumulative distribution function $F(x)$, called the cumulative entropy $(\mathrm{CE})$ and its dynamic version which is analogous to $\mathrm{CRE}$, as follow

$$
\begin{gathered}
\bar{\xi}(X)=-\int_{0}^{\infty} F(x) \log F(x) d x, \\
\bar{\xi}(X ; t)=-\int_{0}^{t} \frac{F(x)}{F(t)} \log \frac{F(x)}{F(t)} d x .
\end{gathered}
$$

Analogous to the Tsallis entropy measure (1.3), Sati and Gupta [33] proposed a cumulative Tsallis entropy (CTE) measure and its dynamic version, parallel to the cumulative entropy $(C E)$, which are given as

$$
\bar{\xi}_{\alpha}(X)=\frac{1}{(1-\alpha)}\left(\int_{0}^{\infty} F^{\alpha}(x) d x-E(X)\right),
$$


and

$$
\bar{\xi}_{\alpha}(X ; t)=\frac{1}{(1-\alpha)}\left(\int_{0}^{t} \frac{F^{\alpha}(x) d x}{F^{\alpha}(t)}-E\left(\tilde{X}_{t}\right)\right), t \geq 0
$$

respectively. For more details and application of these information measures refer to Kumar [17]. When $\alpha \longrightarrow 1$ (5.6) and (5.7) reduces to cumulative entropy (5.4) and past cumulative entropy (5.5) respectively.

Sankaran and Sunoj [32] have considered the quantile version of cumulative past entropy (5.5), which is defined as

$$
\bar{\xi}(u)=\bar{\xi}(X ; Q(u))=\frac{\log u}{u} \int_{0}^{u} p q(p) d p-u^{-1} \int_{0}^{u} p(\log p) q(p) d p .
$$

In analogy to (2.8), we propose a past cumulative Tsallis quantile entropy (PCTQE) that computes the uncertainty related to past. It is defined as

$$
\bar{\xi}_{\alpha}(u)=\bar{\xi}_{\alpha}(X ; Q(u))=\frac{1}{(1-\alpha)}\left(\frac{1}{u^{\alpha}} \int_{0}^{u} p^{\alpha} q(p) d p-\frac{1}{u} \int_{0}^{u} p q(p) d p\right) .
$$

When $u \longrightarrow 1$, (5.9) reduces to $\bar{\xi}_{\alpha}=\frac{1}{(1-\alpha)}\left(\int_{0}^{1} p^{\alpha} q(p) d p-\int_{0}^{1} p q(p) d p\right)$, a quantile version of CTE. Equation (5.9) can be written as

$$
\begin{gathered}
(1-\alpha) \bar{\xi}_{\alpha}(u)=\left(\frac{1}{u^{\alpha}} \int_{0}^{u} p^{\alpha} q(p) d p-\bar{M}(u)\right), \\
u^{\alpha}\left((1-\alpha) \bar{\xi}_{\alpha}(u)+\bar{M}(u)\right)=\int_{0}^{u} \frac{p^{\alpha-1}}{\bar{K}(p)} d p,
\end{gathered}
$$

where (5.10) and (5.11) are the expressions of $\bar{\xi}_{\alpha}(u)$ in terms of the mean inactivity quantile function $\bar{M}(u)$ and reversed hazard quantile function $\bar{K}(u)$, respectively. Differentiating (5.9) with respect to $u$ both sides, and after some algebraic simplification we get

$$
\bar{M}(u)=\alpha \bar{\xi}_{\alpha}(u)+u \bar{\xi}_{\alpha}^{\prime}(u) .
$$

Like $\bar{\xi}_{\alpha}(u)$, equation (5.12) provides a simple relationship between $\bar{\xi}_{\alpha}(u)$ and $\bar{M}(u)$ and thus $\bar{\xi}_{\alpha}(u)$ uniquely determines the quantile density function $q(u)$.

To find an upper bound to $\bar{\xi}_{\alpha}(X ; Q(u))$, we state the following theorem without proof.

Theorem 5.1. If past cumulative Tsallis quantile entropy (PCTQE) $\bar{\xi}_{\alpha}(u)$ is increasing (decreasing) in $u$, if and only if

$$
\bar{\xi}_{\alpha}(u) \leq(\geq) \frac{\bar{M}(u)}{\alpha}, \text { for } 0 \leq u \leq 1 .
$$

Example 4. If $X$ be a random variable having the Tukey lambda distribution with the quantile fuction $Q(u)=\frac{u^{\lambda}-(1-u)^{\lambda}}{\lambda}, 0 \leq u \leq 1$; define for all non-zero lambda values. Then past cumulative Tsallis quantile entropy (5.9) for Tukey lambda distribution is given as

$$
(1-\alpha) \bar{\xi}_{\alpha}(u)=\left[\frac{1}{u^{\alpha}} \int_{0}^{u} p^{\alpha}\left\{p^{\lambda-1}+(1-p)^{\lambda-1}\right\} d p-\frac{1}{u} \int_{0}^{u} p\left\{p^{\lambda-1}+(1-p)^{\lambda-1}\right\} d p\right],
$$

which gives

$$
\bar{\xi}_{\alpha}(u)=\frac{u^{\lambda}}{(\alpha+\lambda)(1+\lambda)}+\frac{1}{(1-\alpha)}\left(\frac{\beta_{u}(\alpha+1, \lambda)}{u^{\alpha}}-\frac{\beta_{u}(2, \lambda)}{u}\right),
$$


where $\beta_{u}(a, b)$ are known as the incomplete beta functions. When $u \rightarrow 1,(5.13)$ gives

$$
\bar{\xi}_{\alpha}=\frac{1}{(1-\alpha)}\left(\beta(\alpha+1, \lambda)-\frac{1}{\alpha+\lambda}-\frac{1}{\lambda}\right)
$$

the expression of past cumulative Tsallis quantile entropy for Tukey Lambda distribution.

Let $X$ have the power distribution with cumulative distribution function (cdf)

$$
F(x)=\left(\frac{x}{a}\right)^{b}, 0<x<a, b>0 .
$$

In the following theorem we show that the power distribution can be characterized in terms of $\bar{\xi}_{\alpha}(X ; Q(u))$.

Theorem 5.2. Let $X$ be a nonnegative continuous random variable with quantile function $Q(u)$ and mean residual quantile function $M(u)$. The relationship

$$
\bar{\xi}_{\alpha}(u)=C \bar{M}(u)
$$

where $C=\frac{b}{(\alpha b-b+1)}$ is constant, holds for all $u$, if and only if, $X$ has power distribution function. The proof is similar to that of Theorem 3.1 and hence is omitted.

Next, we extend the result (5.14) to a more general case taking $C$ as a function of $u$. We state the following result:

Theorem 5.3. Let $X$ be a nonnegative continuous random variable with mean inactivity quantile function $\bar{M}(u)$ and PCTQE $\bar{\xi}_{\alpha}(u)$ given by

$$
\bar{\xi}_{\alpha}(u)=C(u) \bar{M}(u) \text { for } u \geq 0,
$$

then

$$
\bar{M}(u)=\frac{\exp \left(\int_{0}^{u} \frac{d u}{(u C(u)}\right)}{u^{\alpha} C(u)} .
$$

Proof. Substituting (5.15) in (5.12), we get

$$
\bar{M}(u)=\alpha C(u) \bar{M}(u)+u \bar{\xi}_{\alpha}^{\prime}(u) .
$$

Differentiating (5.15) with respect to $u$ and substituting for $\bar{\xi}_{\alpha}^{\prime}(u)$, from (5.17) we obtain

$$
\frac{\bar{M}^{\prime}(u)}{\bar{M}(u)}=\frac{1}{u C(u)}-\frac{\alpha}{u}-\frac{C^{\prime}(u)}{C(u)} .
$$

Integrating with respect to $u$ both side between 0 to $u$ in the above expression and simplifying, we obtain

$$
\bar{M}(u)=\frac{\exp \left(\int_{0}^{u} \frac{d u}{(u C(u)}\right)}{u^{\alpha} C(u)} .
$$

This proves the result.

Example 5. Let $C(u)=a u+b$, and $a, b \geq 0$. From (5.15), we obtain the general model with mean inactivity quantile function

$$
\bar{M}(u)=\frac{1}{u^{\alpha}(a u+b)}\left(\frac{u b}{(a u+b)}\right)^{\frac{1}{b}} .
$$

Further for if $a=0,(5.18)$ gives the characterization result given by the Theorem 5.2. 


\section{$6 \quad$ Weighted Tsallis Quantile Entropy}

The Shannon entropy (1.1) is not scale invariant but it is a shift-independence measure, because $H(C+X)=H(X)$ for some constant $C$. However, in some practical situations, such as reliability or neurobiology, a shift-dependent measure of uncertainty is desirable. Considering this, Belis and Guiasu [4] and later Di Crescenzo and Longobardi [6] considered the notion of weighted entropy. Which is defined as

$$
H^{w}(X)=-\int_{0}^{\infty} x f(x) \log f(x) d x
$$

The factor $x$ inside the integral on the right-hand side of above expression yields a length-biased shift-dependent information measure assigning greater importance to larger values of the random variable $X$. The use of weighted entropy are also motivated by the need arising in various communication and transmission problems of expressing the usefulness of events with an information measure, refer to Kumar and Taneja [19]. Das [5] has studied the importance of weighted generalized entropy for residual and past problems.

Let $X^{w}$ be a weighted random variable corresponding to $X$ with weight function $w(x)$ which is positive for all values of $x \geq 0$. Then the corresponding p.d.f. $f^{w}(x)$ of the random variable $X^{w}$ is given by

$$
f_{w}(x)=\frac{w(x) f(x)}{\mathbb{E}(w(X))}, 0 \leq x<\infty,
$$

with $0<\mathbb{E}(w(X))<\infty$. When $w(x)=x, X^{w}$ is said to be a length biased (or a size biased) random variable. Using $f_{w}(x)$, the corresponding density quantile function is given by

$$
f_{w}(Q(u))=\frac{w(Q(u)) f(Q(u))}{\mu},
$$

where $\mu=\int_{0}^{1} w(Q(p)) f(Q(p)) d(Q(p))=\int_{0}^{1} w(Q(p)) d p$. Therefore, the quantile-based weighted Tsallis entropy is of the form

$$
H_{\alpha}^{w}(Q(u))=\frac{1}{1-\alpha}\left(\frac{1}{\mu^{\alpha}} \int_{0}^{1}[w(Q(p))]^{\alpha}(q(p))^{1-\alpha} d p-1\right) .
$$

In case of length (size) biased random variable the above expression known as length biased weighted Tsallis quantile entropy, which is given as

$$
H_{\alpha}^{L}(Q(u))=\frac{1}{1-\alpha}\left(\frac{1}{\mu^{\alpha}} \int_{0}^{1} Q(p)^{\alpha}(q(p))^{1-\alpha} d p-1\right) .
$$

For some specific univariate continuous distributions, the expression (6.2) is evaluated as given below in Table 6.1.

Now, we consider a random variable $X_{E}$ with density function $f_{E}(x)=\frac{\bar{F}(x)}{\mu}$, with $\mu=$ $E(X)<\infty$. Then, $X_{E}$ is called the equilibrium random variable of the original random variable $X$, and its distribution as equilibrium distribution of original random variable. The equilibrium distribution arises as the limiting distribution of the forward recurrence time in a renewal process. We have $f_{E}(Q(u))=\frac{\bar{F}(Q(u))}{\mu}=\frac{1-u}{\mu}$.

Thus quantile density function for equilibrium distribution is given by $q_{E}(u)=\frac{1}{f_{E}(Q(u))}=\frac{\mu}{1-u}$. 


\begin{tabular}{|c|c|c|}
\hline Distribution & Quantile function $Q(u)$ & $H_{\alpha}^{L}(Q(u))$ \\
\hline Uniform & $a+(b-a) u$ & $\frac{1}{1-\alpha}\left\{\frac{\left(b^{1+\alpha}-a^{1+\alpha}\right) 2^{\alpha}}{(a+b)^{\alpha}(b-a)^{\alpha}(1+\alpha)}-1\right\}$ \\
Exponential & $-\lambda^{-1} \log (1-u)$ & $\frac{1}{1-\alpha}\left\{\frac{\lambda^{\alpha-1} \gamma(1+\alpha)}{\alpha^{1+\alpha}}-1\right\}$ \\
Power & $a u^{\frac{1}{b}}$ & $\frac{1}{1-\alpha}\left\{\frac{b^{1-\alpha}(b+1)}{b \alpha+1}-1\right\}$ \\
Pareto-I & $b(1-u)^{-\frac{1}{a}}$ & $\frac{1}{1-\alpha}\left\{\frac{b^{1-\alpha}(a-1)^{\alpha}}{(\alpha a-1)}-1\right\}$ \\
Finite Range & $b\left(1-(1-u)^{\frac{1}{a}}\right)$ & $\frac{1}{1-\alpha}\left\{\frac{\beta(1+\alpha, 1-\alpha+a)}{a^{-\alpha} b^{\alpha-1}}-1\right\}$ \\
Log-Logestic & $\frac{1}{a}\left(\frac{u}{(1-u)}\right)^{\frac{1}{b}}$ & $\frac{1}{1-\alpha}\left\{\frac{a^{\alpha} \beta\left(\alpha+\frac{1}{b}, \alpha-\frac{1}{b}\right)}{a^{2 \alpha} b^{\alpha}\left(\beta\left(1+\frac{1}{b}, 1-\frac{1}{b}\right)\right)^{\alpha}}-1\right\}$ \\
Weibull & $\frac{\left(-\frac{1}{a} \log (1-u)\right)^{\frac{1}{b}-1}}{a b(1-u)}$ & $\frac{1}{1-\alpha}\left\{\frac{(a b)^{\alpha-1}\left(\frac{1}{a}\right)^{\frac{1}{b}-1} \gamma\left(\frac{\alpha+1}{b}\right)}{\alpha^{\frac{1+\alpha}{b}}\left(\gamma\left(1+\frac{1}{b}\right)\right)^{\alpha}}-1\right\}$ \\
\hline
\end{tabular}

Table 6.1 Length biased weighted Tsallis quantile entropy $\xi_{\alpha}^{L}(X)$ for some lifetime distributions

From (2.8), the dynamic cumulative residual Tsallis quantile entropy (DCRTQE) for equilibrium distribution is given by

$$
\xi_{\alpha}\left(X_{E} ; Q(u)\right)=\frac{1}{1-\alpha}\left(\frac{1}{(1-u)^{\alpha}} \int_{u}^{1}(1-p)^{\alpha} q_{E}(p) d p-\frac{1}{(1-u)} \int_{u}^{1}(1-p) q_{E}(p) d p\right)
$$

Theorem 6.1. Let $X$ be a nonnegative continuous random variable, with relationship for DCRTQE for equilibrium distribution is given as $\xi_{\alpha}\left(X_{E} ; Q(u)\right)=\frac{\mu}{\alpha}$, if and only if $X$ has equilibrium distribution.

Proof. If part of the theorem is easy to proves, to prove only if part let us assume that

$$
\xi_{\alpha}\left(X_{E} ; Q(u)\right)=\frac{\mu}{\alpha} .
$$

From equation (6.3), we have

$$
\int_{u}^{1}(1-p)^{\alpha} q_{E}(p) d p-(1-u)^{\alpha-1} \int_{u}^{1}(1-p) q_{E}(p) d p=\frac{\mu(1-\alpha)(1-u)^{\alpha}}{\alpha} .
$$

Differentiating it with respect to $u$ both sides, after some simplification we get

$$
\int_{u}^{1}(1-p) q_{E}(p) d p=\mu(1-u) .
$$

Again, differentiating with respect to $u$, we obtain $q_{E}=\frac{\mu}{(1-u)}$, the quantile density function for equilibrium distribution. Hence proved.

The following theorem characterizes power distribution using the $\xi_{\alpha}\left(X_{E} ; Q(u)\right)$. The proof of the result is direct.

Theorem 6.2. The random variable $X$ is distributed power function with $Q(u)=a u^{\frac{1}{b}} ; a, b>0$, holds for all $u$ if and only if satisfies the relationship

$$
(1-\alpha) \xi_{\alpha}\left(X_{E} ; Q(u)\right)=C
$$

where $C$ is constant. 
Remark 2. The mean residual quantile function satisfies the relation $M\left(X_{E} ; Q(u)\right)=\mu$ if and only if $X$ follows equilibrium distribution.

\subsection{Weighted Cumulative Residual Tsallis Entropy)}

When the weight function depends on the length of the component, the resulting distribution is called length biased weighted function. Di Crescenzo and Longobardi [6] have considered a length based shift dependent information measure, related to the differential entropy and also introduced the concept of weighted residual entropy and weighted past entropy. Further, Misagh et al. [22] proposed a weighted information which is based on the CRE, called weighted cumulative residual entropy (WCRE). This measure is defined as

$$
\overline{\xi^{w}}(X)=-\int_{0}^{\infty} x \bar{F}(x) \log \bar{F}(x) d x .
$$

Several authors studied properties of (6.4) and its dynamic version, refer to Kayal and Moharana [13] and Mirali et al. [21]. An important feature of the human visual system is that it can recognize objects in a scale and translation invariant manner. However, achieving this desirable behavior using biologically realistic network is a challenge. Analogous to (6.4), Khammar and Jahanshahi [15] have introduced the concepts of weighted cumulative residual Tsallis entropy (WCRTE), and its residual form, defined as

$$
\xi_{\alpha}^{w}(X)=\frac{1}{1-\alpha}\left(\int_{0}^{\infty} x \bar{F}^{\alpha}(x) d x-1\right), \alpha \neq 0, \alpha>1,
$$

and

$$
\xi_{\alpha}^{w}(X, t)=\frac{1}{1-\alpha}\left(\frac{\int_{t}^{\infty} x \bar{F}^{\alpha}(x) d x}{\bar{F}^{\alpha}(t)}-1\right),
$$

respectively. The factor $x$ in the integral on right-hand side yields a "length-biased " shift dependent information measure assigning greater importance to larger values of the random variable $X$. From (1.8) and (2.1), we propose the quantile version of WCTRE and its residual form of a nonnegative random variable $X$, defined as

$$
\xi_{\alpha}^{w}=\frac{1}{1-\alpha}\left(\int_{0}^{1} Q(u)(1-u)^{\alpha} q(u) d u-1\right),
$$

and

$$
\xi_{\alpha}^{w}(u)=\xi_{\alpha}^{w}(X ; Q(u))=\frac{1}{1-\alpha}\left\{\frac{\int_{u}^{1} Q(p)(1-p)^{\alpha} q(p) d p}{(1-u)^{\alpha}}-1\right\},
$$

respectively. The measure (6.8) may be considered as the dynamic weighted cumulative residual Tsallis quantile entropy (DWCRTQE) measure. An alternative expression for the DWCRTQE in terms of mean residual quantile function $M(u)$ of random variable $X$ is the following

$$
\xi_{\alpha}^{w}(u)=\frac{1}{1-\alpha}\left\{\frac{\int_{u}^{1}(1-p)^{\alpha-1} Q(p) M(p) d p}{(1-u)^{\alpha}}-\frac{\int_{u}^{1}(1-p)^{\alpha-1} Q(p) M^{\prime}(p) d p}{(1-u)^{\alpha}}-1\right\} .
$$

Example 6. Let $X$ follow lambda family of distribution as given in example 2.1, then weighted cumulative residual Tsallis quantile entropy (WCRTQE)(6.7) is given as

$$
\xi_{\alpha}^{w}=\frac{1}{(1-\alpha)}\left\{C^{2} \lambda_{1} \beta\left(2 \lambda_{1}, 1+\alpha\right)+C^{2} \lambda_{2} \beta\left(2 \lambda_{1}+1, \alpha-2 \lambda_{2}\right)-1\right\}
$$


As $\lambda_{1} \longrightarrow 0,(6.9)$ reduces to

$$
\xi_{\alpha}^{w}=\frac{1}{(1-\alpha)}\left(\frac{C^{2} \lambda_{2}}{\alpha-2 \lambda_{2}}-1\right)
$$

corresponding to Pareto II distribution. Also, as $\lambda_{2} \longrightarrow 0,(6.9)$ reduces to

$$
\xi_{\alpha}^{w}=\frac{1}{(1-\alpha)}\left(C^{2} \lambda_{1} \beta\left(2 \lambda_{1}, 1+\alpha\right)\right)
$$

corresponding to Power distribution.

For some well-known univariate continuous families of distributions, the expression (6.8) is evaluated as given below in Table 6.2. 
Table 6.2 DWCRTQE for some well-known families of distributions

\begin{tabular}{|c|c|c|}
\hline Distribution & Quantile function $Q(u)$ & $\xi_{\alpha}^{w}(u)$ \\
\hline Uniform & $a+(b-a) u$ & $\frac{1}{1-\alpha}\left\{\frac{a(b-a)(1-u)}{1+\alpha}+\frac{(b-a)^{2}(1-u)}{1+\alpha}-\frac{(b-a)^{2}(1-u)^{2}}{2+\alpha}-1\right\}$ \\
Exponential & $-\lambda^{-1} \log (1-u)$ & $\frac{1}{1-\alpha}\left\{\frac{\bar{\gamma}_{-\log }(1-u)(2, \alpha)}{\lambda^{2}(1-u)^{\alpha}}-1\right\}$ \\
Power & $a u^{\frac{1}{b}}$ & $\frac{1}{1-\alpha}\left\{\frac{a^{2} \bar{\beta}_{u}\left(\frac{2}{b}, 1+\alpha\right)}{b(1-u)^{\alpha}}-1\right\}$ \\
Pareto-I & $b(1-u)^{-\frac{1}{a}}$ & $\frac{1}{1-\alpha}\left\{\frac{b^{2}(1-u)^{\frac{-2}{a}}}{(\alpha a-2)}-1\right\}$ \\
Folded Cramer & $\frac{u}{\theta(1-u)}$ & $\frac{1}{1-\alpha}\left\{\frac{(1-u)^{-2}}{\theta^{2}(\alpha-2)}-\frac{(1-u)^{-1}}{\theta^{2}(\alpha-1)}-1\right\}$ \\
Generalized Pareto & $\frac{b}{a}\left[(1-u)^{-\frac{a}{a+1}}-1\right\}$ & $\frac{1}{1-\alpha}\left\{\frac{b^{2}(1-u)^{\frac{-2 a}{a+1}}}{a(a \alpha+\alpha-2 a)}-\frac{b^{2}(1-u)^{\frac{-a}{a+1}}}{a(\alpha a+\alpha-a)}-1\right\}$ \\
Finite Range & $b\left(1-(1-u)^{\frac{1}{a}}\right)$ & $\frac{1}{1-\alpha}\left\{\frac{b^{2}(1-u)^{\frac{1}{a}}}{(a \alpha+1)}-\frac{b^{2}(1-u)^{\frac{2}{a}}}{(\alpha a+2)}-1\right\}$ \\
Log-Logestic & $\frac{1}{a}\left(\frac{u}{(1-u)}\right)^{\frac{1}{b}}$ & $\frac{1}{1-\alpha}\left\{\frac{\bar{\beta}_{u}\left(\frac{2}{b}, \alpha-\frac{2}{b}\right)}{a^{2} b(1-u)^{\alpha}}-1\right\}$ \\
Weibull & $\left(-\frac{1}{a} \log (1-u)\right)^{\frac{1}{b}}$ & $\frac{1}{1-\alpha}\left\{\frac{\left(\frac{1}{a}\right)^{\frac{2}{b}-1} \bar{\gamma}_{-\log (1-u)}\left(\frac{2}{b}, \alpha\right)}{a b(1-u)^{\alpha}}-1\right\}$ \\
Rayleigh & $\left(-\frac{1}{a} \log (1-u)\right)^{\frac{1}{2}}$ & $\frac{1}{1-\alpha}\left\{\frac{1}{2 a \alpha}-1\right\}$ \\
Gompertz & $\frac{1}{\log C}\left\{1-\frac{1}{\log C \log (1-u)}{ }^{B}\right\}$ & $\frac{1}{1-\alpha}\left\{\frac{1}{\alpha B \log C}+\frac{\bar{\gamma}_{-\log (1-u)}(2, \alpha)}{B^{2}(1-u)^{\alpha}}-1\right\}$ \\
Govindarajulu's & $a\left((b+1) u^{b}-b u^{b+1}\right)$ & $\frac{1}{1-\alpha}\left\{\frac{a^{2} b(b+1)^{2} \bar{\beta}_{u}(2 b, \alpha+2)}{(1-u)^{\alpha}}-\frac{a^{2} b^{2}(b+1) \bar{\beta}_{u}(2 b+1, \alpha+2)}{(1-u)^{\alpha}}-1\right\}$ \\
\hline
\end{tabular}

where $\bar{\gamma}_{x}(a, b)$,and $\bar{\beta}_{u}(a, b)$ known as the incomplete gamma and incomplete beta function defined as $\bar{\gamma}_{x}(a, b)=\int_{x}^{\infty} y^{a-1} e^{-b y} d y$, and $\bar{\beta}_{x}(a, b)=\int_{x}^{1} y^{a-1}(1-y)^{b-1} d y, a, b>0, x>0$ respectively.

The following lemma gives the properties of $\xi_{\alpha}^{w}(X ; Q(u))$. It shows that $\xi_{\alpha}^{w}(X ; Q(u))$ is a shift dependent measure.

Lemma 6.1. If $Y=a X+b$, with $a>0$ and $b \geq 0$, then

$$
\xi_{\alpha}^{w}(Y ; Q(u))=\frac{a^{2}+a b-1}{1-\alpha}+a^{2} \xi_{\alpha}^{w}(X ; Q(u))+a b \xi_{\alpha}^{w}(X ; Q(u)) .
$$

In order to provide some characterization results for DWCRTE of a nonnegative random variable $X$. Let us define the quantile version of weighted mean residual lifetime (WMRL), as follows

$$
M^{w}(u)=m^{w}(Q(u))=\frac{\int_{u}^{1}(1-p) Q(p) q(p) d p}{1-u},
$$

here $m^{w}(t)=\frac{\int_{t}^{\infty} x \bar{F}(x) d x}{\bar{F}(t)}$ is the WMRL of random variable $X$. In the following theorem, we characterize Rayleigh distribution using a relationship between DWCRTE and quantile based WMRL.

Theorem 6.3. Let $X$ be an absolutely continuous random variable. Then the relation

$$
(1-\alpha) \xi_{\alpha}^{w}(X ; Q(u))=\frac{M^{w}(u)}{\alpha}-1,
$$

holds if and only if $X$ follows the Rayleigh distribution. 
Proof. The quantile based WMRL (6.10), for rayleigh distribution is given as

$$
M^{w}(u)=\frac{1}{2 a(1-u)} \int_{u}^{1}\left(\frac{-1}{a} \log (1-p)\right)^{\frac{1}{2}}\left(\frac{-1}{a} \log (1-p)\right)^{\frac{-1}{2}} d p=\frac{1}{2 a} .
$$

The weighted cumulative residual Tsallis quantile entropy (6.10), for Rayleigh distribution is

$$
\xi_{\alpha}^{w}(X ; Q(u))=\frac{1}{1-\alpha}\left(\frac{1}{2 a \alpha}-1\right) .
$$

This proves the if part of the Theorem. To prove only if part, let (6.11) holds. Then

$$
\alpha(1-u) \int_{u}^{1}(1-p)^{\alpha} Q(p) q(p) d p=(1-u)^{\alpha} \int_{u}^{1}(1-p) Q(p) q(p) d p .
$$

Differentiating both sides with respect to $u$, we have

$$
\begin{aligned}
& \alpha \int_{u}^{1}(1-p)^{\alpha} Q(p) q(p) d p+\alpha(1-u)^{\alpha+1} Q(u) q(u) \\
&=\alpha(1-u)^{\alpha-1} \int_{u}^{1}(1-p) Q(p) q(p) d p+(1-u)^{\alpha+1} Q(u) q(u),
\end{aligned}
$$

after some algebraic simplification, this gives

$$
\frac{\alpha}{(1-u) Q(u) q(u)}\left(\frac{1}{(1-u)^{\alpha}} \int_{u}^{1}(1-p)^{\alpha} Q(p) q(p) d p-\frac{1}{1-u} \int_{u}^{1}(1-p) Q(p) q(p) d p\right)=1-\alpha .
$$

Using (6.8) and (6.10), we get

$$
\frac{\alpha}{(1-u) Q(u) q(u)}\left\{(1-\alpha) \xi_{\alpha}^{w}(X, u)+1-M^{w}(u)\right\}=1-\alpha .
$$

Substituting (6.11) in (6.12), we obtain

$$
\frac{\alpha}{(1-u) Q(u) q(u)}\left(\frac{M^{w}(u)}{\alpha}-M^{w}(u)\right)=1-\alpha,
$$

which leads to

$$
\frac{M^{w}(u)}{(1-u)}=Q(u) q(u)
$$

Again using (2.2), we have

$$
M^{w}(u) K(u)=-Q(u)
$$

Differentiating (6.10) with respect to $u$ both sides, we have

$$
\frac{d M^{w}(u)}{d u}-\frac{M^{w}(u)}{1-u}=-Q(u) q(u)
$$

Substituting (6.13), gives $\frac{d M^{w}(u)}{d u}=0$ or equivalently $M^{w}(u)=k$ (constant). From (6.13) $M^{w}(u)=\frac{Q(u)}{k}$ or equivalently gives the survival function $\bar{F}(x)=\exp \left(-\frac{x}{2 a^{2}}\right)$ of Rayleigh distribution. 
Theorem 6.4. For a nonnegative random variable $X$, the relationship

$$
\xi_{\alpha}^{w}(X ; Q(u))=C,
$$

where $C$ is a constant holds, then $X$ has the Rayleigh distribution.

Proof. The necessary part follows from the Table 6.2. For the sufficiency part, let us assume (6.15) holds. From (6.8) we have

$$
\left\{(1-\alpha) \xi_{\alpha}^{w}(X ; Q(u))+1\right\}(1-u)^{\alpha}=\int_{u}^{1} Q(p)(1-p)^{\alpha} q(p) d p .
$$

Taking derivative with respect to $u$ both sides we have, after some algebraic simplification

$$
(1-\alpha) \xi_{\alpha}^{\prime} \underset{\alpha}{w}(X ; Q(u))-\frac{\alpha\left[(1-\alpha) \xi_{\alpha}^{w}(X ; Q(u))+1\right]}{1-u}=-q(u) Q(u) .
$$

Using (2.2), this gives

$$
(1-\alpha) \xi_{\alpha}^{\prime} \underset{\alpha}{w}(X ; Q(u))-\frac{\alpha\left[(1-\alpha) \xi_{\alpha}^{w}(X ; Q(u))+1\right]}{1-u}=-\frac{Q(u)}{(1-u) K(u)} .
$$

From (6.15), we get $\xi^{\prime} \underset{\alpha}{w}(X ; Q(u))=0$. Substitute this value in the above expression we obtain

$$
\alpha\left[(1-\alpha) \xi_{\alpha}^{w}(X ; Q(u))+1\right] K(u)-Q(u)=0,
$$

which leads to $\frac{K(u)}{Q(u)}=\frac{1}{\alpha[C(1-\alpha)+1]}=2 a$ (constant). Thus $X$ follows Rayleigh distribution with survival function $\bar{F}(x)=\exp \left(-\frac{x}{2 a^{2}}\right)$. Hence, the proof is completed.

\section{Conclusion}

An important generalization of Shannon entropy is the non-additive Tsallis entropy measure which finds justifications in many physical, biological and chemical phenomena. In several clinical studies, particularly when the associated diseases are chronic or/and incurable, it is of great concern to patients to know residual and past lifetime. The present work introduced an alternative approach to Tsallis dynamic (residual and past both) entropy using quantile functions. Also there are several uncertainty measures that play a central role in understanding and describing reliability. Most of these information measures do not take into account the values of a random variable. They consider only its probability density. We describing this we proposed the shift dependent quantile-based Tsallis entropy and studied it, with properties similar to those of the legacy entropies.

\section{Acknowledgement}

The first author wishes to acknowledge the Science and Engineering Research Board (SERB), Government of India, for the financial assistance (Ref. No. ECR/2017/001987) for carrying out this research work. 
Some results on quantile-based dynamic survival and failure Tsallis entropy 341

\section{References}

[1] M. Abbasnejad, N. R. Arghami, S. Morgenthaler and G. R. Mohtashami Borzadaran, On the dynamic survival entropy, Statistics and Probability Letters, 80(2010), 1962-1971.

[2] M. Asadi, N. Ebrahimi and E. S. Soofi, Dynamic generalized information measures, Statistics and Probability Letters, 71 (2005), 85-98.

[3] M. Asadi and Y. Zohrevand, On the dynamic cumulative residual entropy, Journal of Statistical Planning and Inference, 137(6), (2007), 1931-1941.

[4] M. Belis and S. Guiasu, A qualitative measure of information in cybernatic systems, IEEE Transactions on Information Theory, 4 (1968), 593-594.

[5] S. Das, On weighted generalized entropy, Communications in Statistics-Theory and Methods, 46(12)(2017), 5707-5727.

[6] A. Di Crescenzo, M. Longobardi, On weighted residual and past entropies, Scientiae Mathematicae Japonicae, 64 (2006), 255-266.

[7] A. Di Crescenzo, M. Longobardi, On cumulative entropies, Journal of Statistical Planning and Inference, 139(12)(2009), 4072-4087.

[8] N. Ebrahimi, How to measure uncertainty in the residual lifetime distribution, Sankhyã Ser. A, 58 (1996), 48-56.

[9] W. Gilchrist, Statistical modelling with quantile functions, Chapman and Hall, CRC, Boca Raton, FL, 2000.

[10] V. H. Hamity and D. E. Barraco, Generalized nonextensive thermodynamics applied to the cosmical background radiation in Robertson-Walker universe, Physical Review Letter, 76 (1996), 4664-4666.

[11] R. K. S. Hankin and A. Lee, A new family of nonnegative distributions, Australian and New Zealand Journal of Statistics, 48(2006) 67-78.

[12] J. Havrda and F. Charvat, Quantification method of classification process: concept of structural $\alpha$-entropy, Kybernetika, 3 (1967), 30-35.

[13] S. Kayal and R. Moharana, On weighted cumulative residual entropy, Journal of Statistics and Management Systems, 20 (2017), 153-173.

[14] S. Kayal and M. R. Tripathy, A quantile-based Tsallis- $\alpha$ divergence, Physica A: Statistical Mechanics and its Applications, 492 (2018), 496-505.

[15] A. H. Khammar and S. M. A. Jahanshahi, On weighted cumulative residual Tsallis entropy and its dynamic version, Physica -A Statistical Mechanics and its Applications, 491 (2018), 678-692.

[16] A. S. Krishanan, S. M. Sunoj and P. G. Sankaran, Quantile-based reliability aspects of cumulative Tsallis entropy in past lifetime, Metrika, 82(1) (2018), 17-38.

[17] V. Kumar, Tsallis entropy measure and k-record values, Physica- A Statistical Mechanics and its Applications, 462(2016), 667-673. 
[18] V. Kumar and Rekha, Quantile approach of dynamic generalized entropy (divergence) measure, Statistica, 78(2)(2018), 105-126.

[19] V. Kumar and H. C. Taneja, On length biased dynamic measure of past inaccuracy, Metrika, 75 (1) (2012), 73-84.

[20] V. Kumar and H. C. Taneja, A generalized entropy-based residual lifetime distributions, International Journal of Biomathematics, 4(2) (2011), 171-184.

[21] M. Mirali, S. Baratpour and V. Fakoor, On weighted cumulative residual entropy, Communications in Statistics-Theory and Methods, 46(6) (2017), 2857-2869.

[22] F. Misagh, Y. Panahi, G.H. Yari and R. Shahi, Weighted cumulative entropy and its estimation. In: 2011 IEEE International Conference on Quality and Reliability (ICQR), (2011), doi:10.1109/ICQR.2011.6031765

[23] N. U. Nair, P. G. Sankaran and N. Balakrishnan, Quantile-based reliability analysis, New York: Springer, 2013.

[24] N. U. Nair and P. G. Sankaran, Quantile-based reliability analysis, Communications in Statistics Theory and Methods, 38(2) (2009), 222-232.

[25] A. K. Nanda and P. Paul, Some results on generalized residual entropy, Information Sciences, 176(1) (2006), 27-47.

[26] A. K. Nanda, P. G. Sankaran and S.M. Sunoj, Residual Renyi entropy : a quantile qpproach, Statistics and Probability Letters, 85 (2014), 114-121.

[27] G. Rajesh and S.M. Sunoj, Some properties of cumulative Tsallis entropy of order $\alpha$, Statistical papers, (2016), 1-11.

[28] M. Rao, Y. Chen, B. C. Vemuri and F. Wang, Cumulative residual entropy: a new measure of information, IEEE Transactions on Information Theory, 50(6) (2004), 1220-1228.

[29] M. Rao, More on a new concept of entropy and information, Journal of Theoretical Probability, 18 (2005), 967-981.

[30] S. Tong, A. Bezerianos, J. Paul, Y. Zhu and N. Thakor, Nonextensive entropy measure of EEG following brain injury from cardiac arrest, Physica A: Statistical Mechanics and its Applications, 305(3-4) (2002), 619-628.

[31] C. Tsallis, Possible generalization of Boltzmann-Gibbs statistics, Journal Statistical Physics, 52(1-2) (1988), 479-487.

[32] P. G. Sankaran and S.M. Sunoj, Quantile-based cumulative entropies, Communications in Statistics Theory and Methods, 46(2) (2017), 805-814.

[33] M. M. Sati and N. Gupta, Some characterization results on dynamic cumulative residual Tsallis entropy, Journal of probability and statistics, (2015), Article ID 694203, 8 Pages, http://dx.doi.org/10.1155/2015/694203.

[34] C. E. Shannon, A mathematical theory of communication, Bell System Technical Journal, 27 (1948), 379-423. 
[35] S. M. Sunoj and P. G. Sankaran, Quantile-based entropy function, Statistics and Probability Letters, 82 (2012), 1049-1053.

[36] S. M. Sunoj, A. S. Krishnan and P. G. Sankaran, A quantile-based study of cumulative residual Tsallis entropy measures, Physica A: Statistical Mechanics and its Applications, 494 (2017), 410-421.

[37] S. M. Sunoj, P. G. Sankaran and A. K. Nanda, Quantile-based entropy function in past lifetime, Statistics and Probability Letters, 83(1) (2013), 366-372.

[38] F. Wang and B. C. Vemuri, Non-Rigid multimodal image registration using cross-cumulative residual entropy, International Journal of Computer Vision, 74(2) (2007), 201-215.

[39] M. Yu, C. Zhanfang, and Z. Hongbiao, Research of automatic medical image segmentation algorithm based on Tsallis entropy and improved PCNN., IEEE proceedings on ICMA, (2009), 1004-1008.

Rekha Rani Department of Applied Sciences, UIET, M. D. University, Rohtak-124001, India.

E-mail: rekha02647@gmail.com

Vikas Kumar Department of Applied Sciences, UIET, M. D. University, Rohtak-124001, India.

E-mail: vikasror.mdu@gmail.com

Nirdesh Singh Department of Electrical Engineering, Deenbandhu Chhotu Ram University of Science and Technology, Murthal, Sonipat-131039, India.

E-mail: singh.niru28@gmail.com 